\title{
Alternative dosing of dual PI3K and MEK inhibition in cancer therapy
}

Elina Jokinen, Niina Laurila and Jussi P Koivunen*

\begin{abstract}
Background: PI3K/AKT/mTOR and RAS/RAF/MEK/ERK pathways are thought to be the central transducers of oncogenic signals in solid malignancies, and there has been a lot of enthusiasm for developing inhibitors of these pathways for cancer therapy. Some preclinical models have suggested that combining inhibitors of both parallel pathways may be more efficacious, but it remains unknown whether dual inhibition with high enough concentrations of the drugs to achieve meaningful target inhibition is tolerable in a clinical setting. Furthermore, the predictive factors for dual inhibition are unknown.

Methods: Non-small cell lung cancer (NSCLC) cell lines $(n=12)$ with the most frequent oncogenic backgrounds ( $K$-Ras mut $n=3$, EGFR mut $n=3$, ALK translocated $n=3$, and triple-negative $n=3$ ) were exposed to PI3K inhibitors (ZSTK474, Pl-103) or MEK inhibitor (Cl-1040) alone or in combination and analysed with an MTS growth/cytotoxicity assay and statistically by combination index analysis. The activity of the intracellular signaling pathways in response to the inhibitor treatments was analysed with a western blot using phospho-specific antibodies to AKT, ERK1/2, S6, and $4 \mathrm{E}-\mathrm{BPI}$. For the differential dosing schedule experiments, additional breast and colon cancer cell lines known to be sensitive to dual inhibition were included.

Results: Two of the 12 NSCLC cell lines tested, H3122 (ALK translocated) and H1437 (triple-negative), showed increased cytotoxicity upon dual MEK and PI3K inhibition. Furthermore, MDA-MB231 (breast) and HCT116 (colon), showed increased cytotoxicity upon dual inhibition, as in previous studies. Activation of parallel pathways in the dual inhibition-sensitive lines was also noted in response to single inhibitor treatment. Otherwise, no significant differences in downstream intracellular pathway activity ( $\mathrm{S} 6$ and 4E-BPI) were noted between PI3K alone and dual inhibition other than the increased cytotoxicity of the latter. In the alternative dosing schedules two out of the four dual inhibition-sensitive cell lines showed similar cytotoxicity to continuous PI3K and short (15min) MEK inhibition treatment.

Conclusions: Therapy with a dual PI3K and MEK inhibitor combination is more efficient than either inhibitor alone in some NSCLC cell lines. Responses to dual inhibition were not associated with any specific oncogenic genotype and no other predictive factors for dual inhibition were noted. The maximal effect of the dual PI3K and MEK inhibition can be achieved with alternative dosing schedules which are potentially more tolerable clinically.
\end{abstract}

Keywords: Non-small cell lung cancer, PI3K inhibition, MEK inhibition

\footnotetext{
* Correspondence: jussi.koivunen@ppshp.fi

Department of Medical Oncology and Radiotherapy, Oulu University Hospital, Oulu PB22 90029 OYS, Finland
} 


\section{Background}

Constitutive activation of oncogenic pathways occurs in cancers with very high frequency, and this is thought to be a central factor behind the hallmarks of cancer phenotypes, such as cycle progression, inhibition of apoptosis and metabolic reprogramming. The PI3K-AKT and RAS-RAFMEK-ERK pathways are thought to play a central role in transmitting these oncogenic signals. Frequent cancerassociated genetic alterations such as receptor mutations or amplifications, mutations in intermediate signal transducers such as Ras, Raf or PI3KCA and inactivation of certain tumor suppressors such as PTEN lead to constitutive activation of these pathways [1].

The high frequency of cancer-associated genetic alterations causing constitutive activation of PI3K-AKT and RAF-MEK-ERK and the addiction of cancer cells to their signals have led to enthusiasm for developing inhibitors of these pathways. In view of the central role of such pathways in transmitting upstream oncogenic signals, their inhibition could be an effective therapy for various cancer genotypes. Some cancer genotypes have been identified in preclinical studies as responders to specific inhibitors of the pathways. HER2 amplified breast cancers have been shown to respond to PI3K inhibitors [2], while B-Raf mutant melanomas [3] and triple-negative breast cancers are repressed by MEK inhibitors [4]. The effectiveness of single pathway inhibition could be suppressed by de novo dependence on multiple signaling pathways or feedback activation of other signaling pathways in response to the inhibition of a single pathway $[2,5]$. This has led to studies combining PI3K or AKT and MEK inhibitors. Dual inhibition has shown increased efficiency in various cancer genotypes in pre-clinical studies $[2,4,6,7]$ and numerous early-phase clinical studies are in progress. Clinical studies have shown the simultaneous inhibition of multiple pathways to be in all probability more toxic than inhibition of a single pathway, and no optimal dose has been established.

PI3K-mTOR inhibitors may be divided into PI3K inhibitors (such as ZSTK474), dual PI3K-mTOR inhibitors (such as PI-103) and mTOR inhibitors (rapalogs). Rapalog mTOR inhibitors are known to induce IRS-1-mediated, upstream feedback activation of PI3K-AKT [8], which is thought to be important for the limited clinical efficiency of the therapy for most cancers, including NSCLC. PI3K and PI3K/mTOR inhibitors should lack such feedback activation and theoretically be more active. Numerous early phase clinical trials are currently testing both single $\mathrm{PI} 3 \mathrm{~K}$ and dual PI3K/mTOR inhibitors, but it is unknown whether either is more efficient, although it is likely that a drug which hits multiple targets will be more toxic in a clinical setting.

Current oncological therapies have modest disease modifying effects in cases of non-small cell lung cancer (NSCLC), even though some disease subgroups responsive to targeted therapy have been identified in recent years. These include EGFR mutant (10-30\% of patients) $[9,10]$ and $A L K$ translocated $(\sim 5 \%)[11,12]$, in which patients are highly responsive to EGFR or ALK tyrosine kinase inhibitors (TKI) $[13,14]$. Furthermore, other major oncogenic disease subgroups include the K-Ras mutant ( 25\% of patients), which is thought to be undruggable with currently available pharmacological agents [15].

We set out here to investigate dual inhibition with PI3K and MEK in non-small cell lung cancer (NSCLC) cell lines of various genotypes. Dual inhibition is shown to be a more effective form of therapy in some cell lines. This study also addresses administration schedules for the inhibitors which may prove less toxic in a clinical setting.

\section{Methods}

\section{Cell lines}

The cell lines used here included NSCLC lines with a K-Ras mutation (A549, H358, H441), EGFR mutation (H1975, HCC827, PC-9), ALK translocation (DFCI032, $\mathrm{H} 2228$, and $\mathrm{H} 3122$ ) and the triple negative genotype (A431, H1437, H1581), a basal-like breast cancer line MDA-MB231 and HCT116, a K-Ras mutant colorectal cell line. The NSCLC cell lines were kind gifts from Dr. Pasi Jänne (Dana-Farber Cancer Institute, Boston, USA), and the breast and colorectal lines from Dr. Peppi Koivunen (Oulu University, Oulu, Finland). The cell lines were cultured in RPMI-1640 supplemented with 5 or $10 \%$ fetal bovine serum and $100 \mathrm{IU} / \mathrm{ml}$ penicillin and streptomycin. All the cell culture reagents were purchased from HyClone (Logan, UT).

\section{Inhibitors}

The following inhibitors were used: CI-1040, PI-103, ZSTK474 (Alexis Biochemicals; Lausen, Switzerland), and TAE684 (a kind gift from Dr. Nathanael Gray, Dana-Farber Cancer Institute, Boston, USA). All the inhibitors were dissolved in DMSO to a final concentration of $10 \mathrm{mM}$ and stored at $-20^{\circ} \mathrm{C}$. The drug solutions for the experiments were prepared from a $10 \mathrm{mM}$ stock solution immediately before use. MEK inhibitor CI-1040 (PD-184352), a specific small-molecule drug that inhibits MEK1/MEK2, is thought to act as an allosteric inhibitor of MEK, since it is known not to compete with the binding of either ATP or protein substrates. CI-1040 blocks ERK phosphorylation and inhibits the growth of multiple human tumor cell lines and tumor growth in xenograft models. It has been shown that the inhibitory effect of CI-1040 on cell growth is rapidly reversed after it is removed from the growth medium [16]. ZSTK474 is a small-molecule PI3K inhibitor which has shown to be a potential antitumor agent against a human cancer xenograft in vivo with no toxicity to any critical organs [17]. It inhibits all four PI3K isoforms, most strongly 
PI3K $\delta$, by competing with the binding of ATP to the ATPbinding-pocket of the protein. In addition, the molecule is significantly specific to PI3K, since even when administered at high concentrations it only weakly inhibits the mTOR complex, which contains a conserved PI3K domain [18]. PI-103 is a pyridofuropyrimidine compound that selectively inhibits PI3K $\alpha$ and mTOR signaling, prevents cell proliferation and invasion, causes G0-G1 cell cycle arrest and reduces tumor growth in glioma xenografts [19]. The inhibitor has also shown significant antitumor potency in NSCLC cell lines [20].

\section{Cytotoxicity/cell growth assay}

Cells were plated onto 96-well plates with three to six parallel wells for each treatment, the experiments being replicated at least three times. The inhibitor treatments were started on the following day, and the plates were developed 72h later using an MTS reagent mix ([3-(4, 5-dimethylthiazol-2-yl)-5-(3-carboxymethoxyphenyl)-2(4-sulfophenyl)-2H-tetrazolium, inner salt], Promega; Madison, WI) supplemented with phenazine methosulfate (Sigma-Aldrich; St. Louis, MO) according to the manufacturer's guidelines. The absorbances were read on a plate reader (Athos Labtec Instruments; Salzburg, Austria) at a wavelength of $488 \mathrm{~nm}$. The data were displayed graphically using GraphPad Prism (GraphPad Software; La Jolla, CA), with the absorbance in the non-treated wells as the reference value (100\%). The combination index (CI) was calculated using Calcusyn software (BIOSOFT, Cambridge, UK), and a 3.3:1 ratio of the PI3K inhibitors to the MEK inhibitor was used in the CI analysis. CI values at ED50 are presented.

\section{Western blot analysis}

The cells were plated onto 6-well plates and treated with the drugs 24-48h later for 6 or $72 \mathrm{~h}$, after which they were lysed in RIPA buffer (1\% Igepal CA-630, $20 \mathrm{mM}$ Tris- $\mathrm{HCl} \mathrm{pH}$ 8.0, $137 \mathrm{mM} \mathrm{NaCl}, 10 \%$ glycerol, $2 \mathrm{mM}$ EDTA, $1 \mathrm{mM}$ sodium orthovanadate, $10 \mu \mathrm{g} / \mathrm{mL}$ Aprotinin, $10 \mu \mathrm{g} / \mathrm{mL}$ Leupeptin, and $10 \mu \mathrm{g} / \mathrm{mL}$ Pepstatin). Protein concentrations were measured using the BioRad Protein Assay (Bio-Rad; Hercules, CA) and the concentrations in individual samples were equalized before adding 3x Laemmli buffer to a final concentration of $1 \mathrm{x}$. Equal amounts of protein were run on $7.5 \%$ SDS-PAGE gels, transferred to PVDF membranes, probed with the antibodies and developed using the ECL chemiluminescence system (Millipore; Billerica, MA) for detection on radiographic films, which were scanned to an electronic format. All the antibodies used were from Cell Signaling Technologies (Danvers, MA): pAKT (S473), AKT, pERK (T202/Y204), ERK, pS6 (Thr389), S6, p4E-BP1 (Thr37/46), 4E-BP1, cleaved PARP. Anti-rabbit HRP conjugated antibody was used as a secondary antibody.

\section{Pathscan analysis}

The PathScan analysis was carried out with the PathScan ${ }^{\circledR}$ RTK Signaling Antibody Array kit (Cell Signaling Technologies, Danvers, MA) according to the manufacturer's guidelines. In brief, cells were plated on plates of diameter $6 \mathrm{~cm}$ and drugged the following day for $24 \mathrm{~h}$. Whole cell lysates were collected, protein concentrations were determined using the Bio-Rad Protein Assay (Bio-Rad, Hercules, CA) and the protein concentrations were equalized. The lysates were applied to nitrocellulose membranes and incubated over night, washed, exposed to the secondary antibodies, developed with ECL and imaged with a Fujifilm LAS-3000 Luminescent Image analyzer and the ImageReader LAS-3000 program. The array target map can be found through the manufacturer's homepage (http://www.cellsignal.com/products/7982.html).

\section{Results}

Dual inhibition of PI3K and MEK in cancer cell lines

The inhibitors used were ZSTK474 (PI3K inhibitor) and PI-103 (PI3K and mTOR inhibitor) and CI-1040 (MEK inhibitor). We first addressed the effects of these inhibitors alone in the NSCLC lines A549 (K-Ras mutant), HCC827 (EGFR mutant) and H3122 (EML4-ALK translocated), representing the three most frequent oncogenic genotypes of the disease, to establish concentration frames for the target inhibition. In the Western blots ZSTK474 at a $3.3 \mu \mathrm{M}$ concentration induced complete downregulation of pAKT, an immediate downstream target of PI3K, while PI-103 induced a similar inhibition at concentrations of 1 to $3.3 \mu \mathrm{M}$ (Figure 1A). pS6 downregulation correlated highly with pAKT downregulation (Figure 1A). The MTS cytotoxicity assay showed a major reduction in the number of viable cells in all the cell lines with similar concentrations of both inhibitors, which were closely correlated with the concentrations inducing complete inhibition of pAKT in Western blot analysis (Figure 1A,C). CI-1040 induced complete inhibition of ERK1/2, an immediate downstream target of MEK, at a $1 \mu \mathrm{M}$ concentration (Figure 1B). Only the H3122 line showed any marked reduction in cell viability in the MTS assays in response to increasing concentrations of the inhibitor, correlating with maximal target inhibition, while the other lines displayed minor changes in viability, except for the $10 \mu \mathrm{M}$ treatment in HCC827, despite the achieving of complete inhibition of pERK1/2 in all the lines tested at $1 \mu \mathrm{M}$ (Figure $1 \mathrm{C}$ ).

Dual inhibition of PI3K and MEK was tested in a panel of NSCLC lines $(\mathrm{n}=12)$ with the K-Ras $(\mathrm{n}=3), E G F R$ $(\mathrm{n}=3), A L K(\mathrm{n}=3)$, or triple-negative $(\mathrm{n}=3)$ oncogenic genotypes. Analogously to the cell lines in the preliminary experiments, all the cell lines tested here showed a major reduction in cell growth in response to the PI3K inhibitors alone, with no significant differences between ZSTK474 or PI-103 (Figure 2A, eight of the twelve lines 


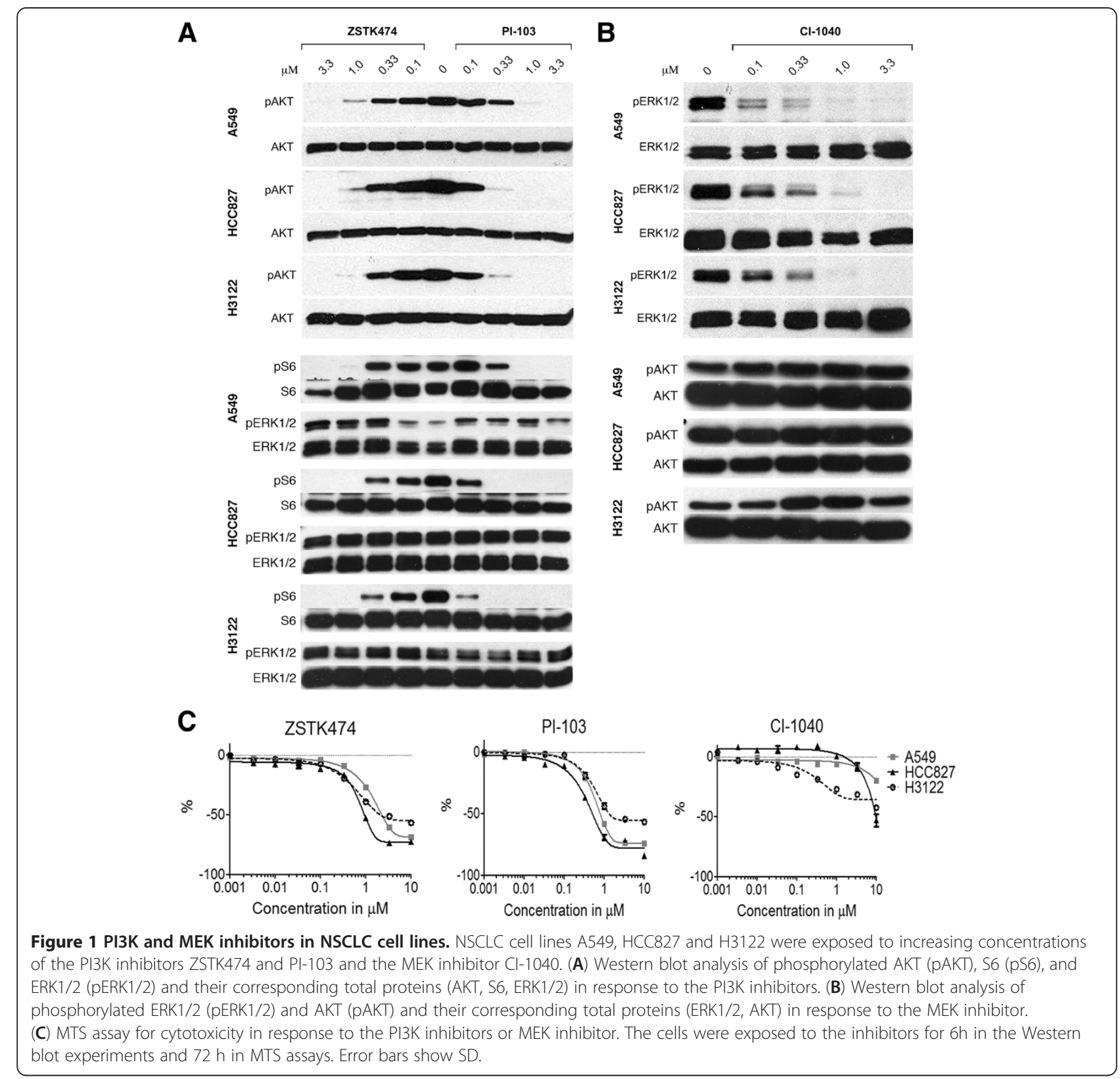

presented graphically). The MEK inhibitor CI-1040 elicited variable responses with the majority of cell lines, showing only minor inhibition of growth or none at all. When the cell lines were exposed to dual, concurrent inhibition of PI3K and MEK, two out of 12 tested cell lines, H3122 and H1437, showed marked additional cytotoxicity compared with treatment with a single agent (Figure 2A). The results were submitted to combination index $(\mathrm{CI})$ analysis and average $\mathrm{CI}$ values were calculated based on combinations of ZSTK474 and PI-103. This analysis grouped the cell lines into three categories: antagonism ( $n=5$, CI 1.10-3.3), nearly additive or slight synergy ( $n=5$, CI $0.7-1.10)$, and synergy or strong synergy
( $n=2$, CI <0.7) (Table 1). Visual assessment of the dual inhibition in MTS curves did not suggest any major antagonism of treatment in any of the lines tested, however, since the combination treatment curves in the cell lines with antagonistic CI values closely followed the single PI3K inhibitor treatment curves (Figure 2A). There was no correlation between the cancer genotypes in responsiveness to the dual inhibition, since an $A L K$ translocated line (H3122) and a triple-negative negative line (H1437) showed synergistic responses to dual inhibition (Figure 2A, Table 1). The NSCLC lines showing synergistic responses to dual inhibition seemed to be more responsive to low concentrations $(<1 \mu \mathrm{M})$ of the 


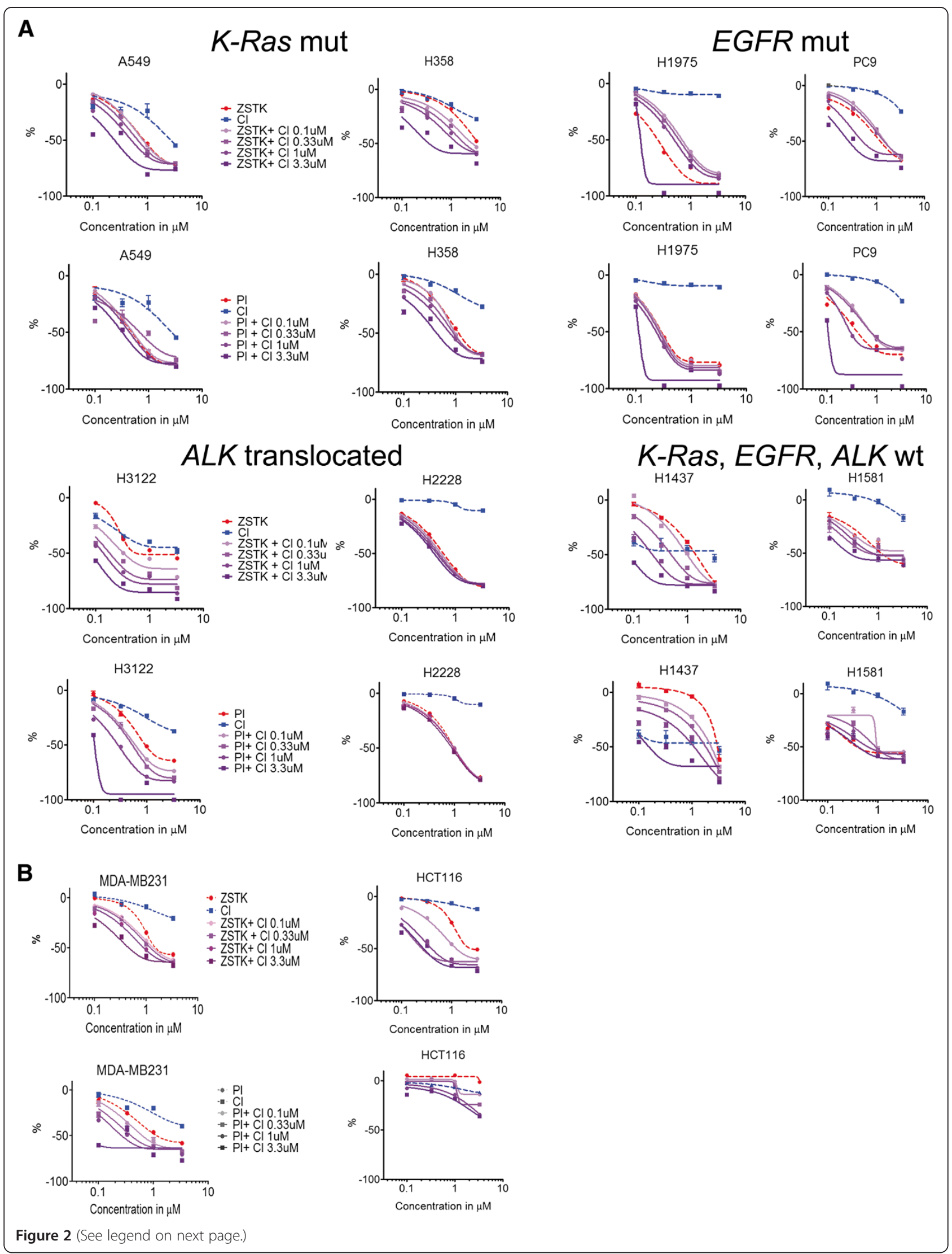


(See figure on previous page.)

Figure 2 PI3K and MEK inhibitors and their combinations in cancer cell lines. The cells were exposed to specific treatments for $72 \mathrm{~h}$ and analyzed with the MTS cytotoxicity assay. The single inhibitor treatments are represented with dashed lines and their combinations with continuous lines. (A) NSCLC cell lines. The cell lines are placed in four groups based on their oncogenic genotype (K-Ras mutant, EGFR mutant, ALK translocated, and triple wild type). (B) Breast cancer line MDA-MB231 and colorectal cancer line HCT116, known from the previous literature to be sensitive to dual PI3K and MEK inhibition. Error bars show SD.

MEK inhibitor alone (Figure 2A). Analogously to the single inhibitor results, the lines sensitive to dual inhibition showed only a minor difference between the activities of the different PI3K inhibitors in combination with the MEK inhibitor.

Based on a literature search [4,7], additional cell lines known to be responsive to dual PI3K and MEK inhibition were studied. MDA-MB231, a basal-like breast cancer line, and HCT116, a K-Ras mutant colorectal line, were exposed to single inhibitors or dual inhibition and analyzed with the MTS assay. As in the previous work, both the cell lines showed synergistic responses to dual inhibition (Figure 2B, Table 1). PI-103 was markedly less effective than ZSTK474 in the HCT116 cell line, while, like all the NSCLC cell lines, MDA-MB231 responded similarly to both PI3K inhibitors (Figure 2B, Table 1). Interestingly, we did not see any differences in target inhibition between ZSTK474 and PI-103 in the HCT116 line (Figure 3A), so that the mechanism of differential efficiency remains unknown.

The lines H3122, H1437, MDA-MB231, and HCT116, which were sensitive to dual inhibition, were further analyzed with Western blot analysis for cleaved PARP, a well-characterized marker of apoptosis. No cleaved PARP was detected in any of the cell lines following the single agent treatments (Figure 4A), but when dual

Table 1 Combination index analysis

\begin{tabular}{|c|c|c|c|c|c|}
\hline Cell line & PI3K inhibitor & $\mathrm{Cl}$ (ED50) & Average $\mathrm{Cl}$ & Range & Significance \\
\hline $\mathrm{H} 1437$ & ZSTK474 & 0,4 & 0,3 & $0,1-0,3$ & Strong synergism \\
\hline $\mathrm{H} 1437$ & $\mathrm{Pl}-103$ & 0,2 & & & \\
\hline $\mathrm{H} 3122$ & ZSTK474 & 0,27 & 0,325 & $0,3-0,7$ & Synergism \\
\hline $\mathrm{H} 3122$ & $\mathrm{Pl}-103$ & 0,38 & & & \\
\hline MDA-MB231 & ZSTK474 & 0,58 & 0,515 & $0,3-0,7$ & Synergism \\
\hline MDA-MB231 & $\mathrm{Pl}-103$ & 0,45 & & & \\
\hline HCT116 & ZSTK474 & 0,4 & 0,635 & $0,3-0,7$ & Synergism \\
\hline HCT116 & $\mathrm{Pl}-103$ & 0,87 & & & \\
\hline DFCI032 & ZSTK474 & 0,61 & 0,71 & $0,7-0,85$ & Moderate synergism \\
\hline DFCl032 & $\mathrm{Pl}-103$ & 0,81 & & & \\
\hline $\mathrm{H} 358$ & ZSTK474 & 0,6 & 0,79 & $0,7-0,85$ & Moderate synergism \\
\hline $\mathrm{H} 358$ & $\mathrm{Pl}-103$ & 0,98 & & & \\
\hline $\mathrm{H} 2228$ & ZSTK474 & 0,87 & 0,885 & $0,85-0,9$ & Slight synergism \\
\hline $\mathrm{H} 2228$ & $\mathrm{Pl}-103$ & 0,9 & & & \\
\hline A549 & ZSTK474 & 0,9 & 0,93 & $0,90-1,10$ & Nearly additive \\
\hline A549 & $\mathrm{Pl}-103$ & 0,96 & & & \\
\hline$\underline{\mathrm{H} 441}$ & ZSTK474 & 1,14 & 1,07 & $0,90-1,10$ & Nearly additive \\
\hline $\mathrm{H} 441$ & $\mathrm{Pl}-103$ & 1 & & & \\
\hline A431 & ZSTK474 & 1,17 & 1,19 & $1,10-1,20$ & Slight antagonism \\
\hline A431 & $\mathrm{Pl}-103$ & 1,21 & & & \\
\hline $\mathrm{HCC} 827$ & ZSTK474 & 0,89 & 1,45 & $1,20-1,45$ & Moderate antagonism \\
\hline HCC827 & $\mathrm{Pl}-103$ & 2,01 & & & \\
\hline PC9 & ZSTK474 & 1,37 & 1,65 & $1,20-1,45$ & Antagonism \\
\hline PC9 & $\mathrm{Pl}-103$ & 1,93 & & & \\
\hline $\mathrm{H} 1518$ & ZSTK474 & 1,2 & 1,72 & $1,45-3,3$ & Antagonism \\
\hline $\mathrm{H} 1518$ & $\mathrm{Pl}-103$ & 2,24 & & & \\
\hline $\mathrm{H} 1975$ & ZSTK474 & 2,3 & 1,745 & $1,45-3,3$ & Antagonism \\
\hline H1975 & Pl-103 & 1,19 & & & \\
\hline
\end{tabular}




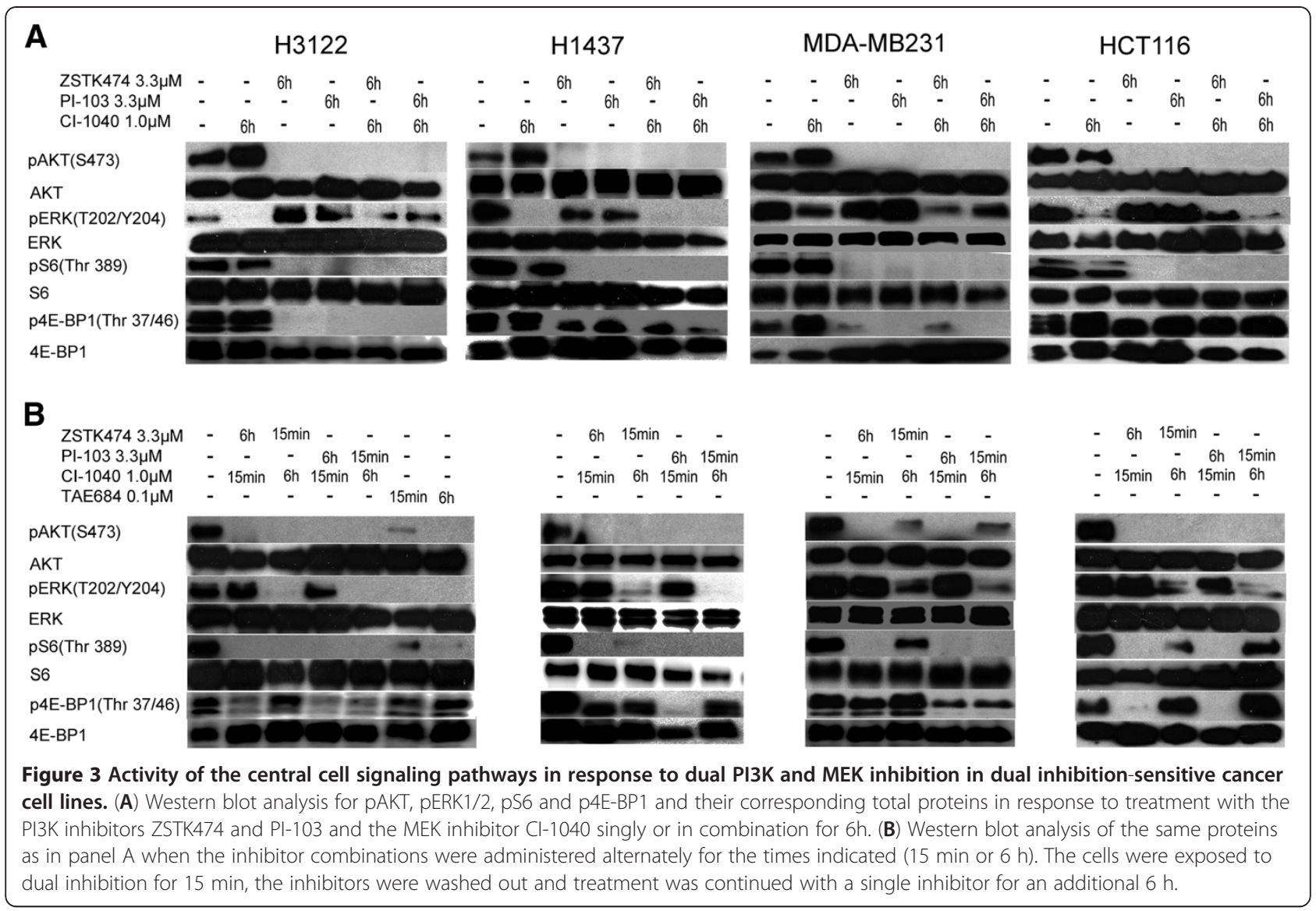

inhibition with either ZSTK474 or PI-103 was administered, marked PARP cleavage was seen in the H3122 line but not in the other lines tested (Figure 4A).

\section{Effect of dual inhibition on cell signaling}

The NSCLC (H3122 and H1437), breast cancer (MDAMB231) and colon cancer (HCT116) lines, which showing major synergy upon dual inhibition, were further studied for cell signaling in response to the inhibitors. All the cell lines downregulated pAKT and its downstream target pS6 completely in response to $6 \mathrm{~h}$ of treatment with the PI3K inhibitor ZSTK474 or PI-103 (3.3 $\mu \mathrm{M})$ (Figure 3A). Downregulation of $\mathrm{p} 4 \mathrm{E}-\mathrm{BP} 1$ was also noted with all the cell lines tested, but it was complete only in the H3122 cell line (Figure 3A). Furthermore, concurrent activation of pERK1/2 was recognized in the H3122, MDA-MB231 and HCT116 cell lines during PI3K inhibitor treatment (Figure 3A). When the cell lines were treated with the MEK inhibitor CI-1040 (1 $\mu \mathrm{M}, 6 \mathrm{~h})$, complete (H3122, H1437) or marked (MDA-MB231, HCT116) downregulation of pERK1/2 was seen (Figure $3 \mathrm{~A}$ ). This was accompanied by upregulation of pAKT in the H3122 and MDA-MB231 lines, but not by upregulation of pS6 or p4E-BP1 (H3122) (Figure 3A). p4E-BP1 was markedly upregulated in the MDA-MB231 line in response to CI1040 treatment (Figure 3A).

When the PI3K and MEK inhibitors were administered simultaneously the inhibition of the targets was similar to that seen with single inhibitor treatment (Figure 3A). Dual inhibition was able to overcome the single inhibitorinduced stimulation of parallel pathway activation (Figure 3A). We were not able to detect any significant difference in the activity of either pS6 or p4E-BP1 following dual inhibitor treatment as compared with the single PI3K inhibitor treatments (Figure 3A).

Further analysis of the dual inhibition of the central RTKs and signaling nodes was carried out with the PathScan Antibody Array, which investigates the phosphorylation status of 28 RTKs and 11 signaling nodes concurrently. Attention was focused on the dual inhibition-sensitive H1437 and MDA-MB231 lines. A low level of RTK activation was noted in untreated cells of both cell lines, H1437 showing some activity with c-MET (Figure 5), while in the signaling nodes, pAKT, S6 and ERK1/2 showed activity in both cell lines and Src activity was also noted in H1437. In the drug-treated cells, ZSTK474 (24 h) was able to inhibit both AKT and S6 phosphorylation, S6 showing a more pronounced effect (Figure 5). Furthermore, ZSTK474 induced a marked 

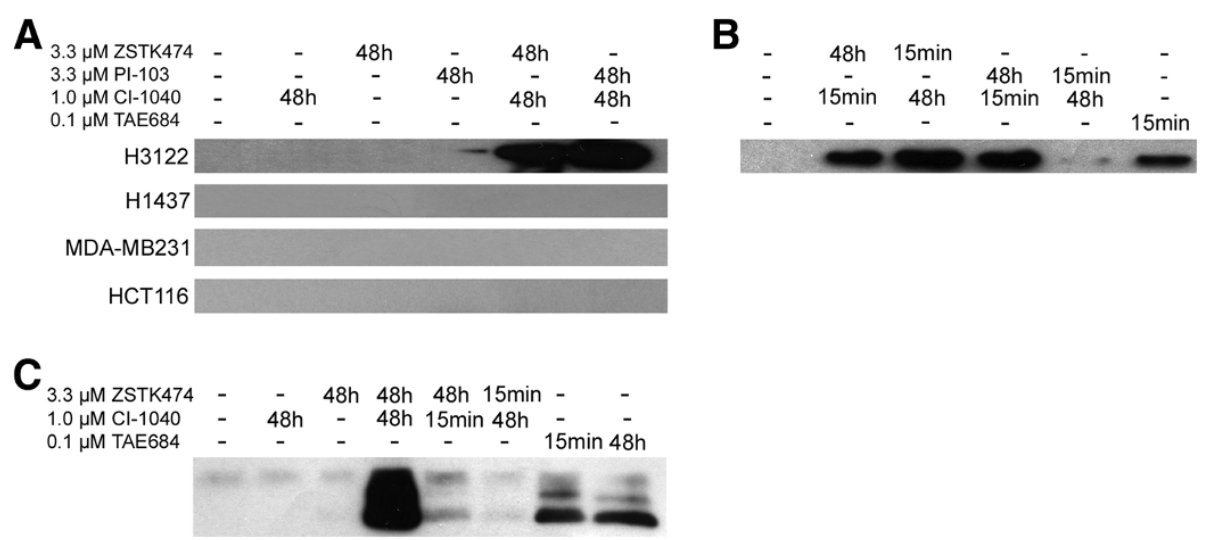

Figure 4 Apoptotic response to dual PI3K and MEK inhibition in dual inhibition-sensitive cancer cell lines. (A) Western blot analysis for cleaved PARP (CPARP) in the H3122, H1437, MDA-MB231 and HCT116 lines treated with the PI3K inhibitors ZSTK474 and PI-103, the MEK inhibitor Cl-1040, or their combinations for 48 h. (B) Western blot analysis for cleaved PARP in the H3122 cell line treated for 15min with dual inhibition and with the single inhibitor indicated for an additional $48 \mathrm{~h}$, or with the ALK inhibitor TAE684 for $15 \mathrm{~min}$. (C) Western blot analysis for cleaved caspase-3 in the H3122 line with indicated treatments and exposure times.

broad feedback RTK activation in the H1437 cell line (Figure 5). CI-1040 (24 h) effects were limited to the inhibition of ERK1/2 activity. When dual inhibition with ZSTK474 and CI-1040 was administered, downregulation of both pAKT/S6 and ERK1/2 was noted, but otherwise no marked difference was evident relative to the single agent treatments (Figure 5). The results suggest specificity of the inhibitors for their targets and the existence of broad feedback activation.

\section{Alternative dosing of dual inhibition}

Even though dual inhibition of PI3K and MEK was identified as an effective form of cancer therapy based on the in vitro models, administration of both drugs at doses inducing major downregulation of the target for long periods of time may be too toxic in a clinical setting. We therefore set out to investigate concurrent administration of PI3K and MEK inhibitors to cell lines sensitive to dual inhibition with alternative dosing schedules. The MTS assays showed that for maximal reduction in the number of living cells in all the lines, dual inhibition needed to be administered for longer periods of time. The therapy was significantly more effective when it was administered throughout the $72 \mathrm{~h}$ experiment as compared with 15 min, $4 \mathrm{~h}$ or $24 \mathrm{~h}$ periods (Figure 6). Interestingly, maximal cytotoxicity was seen in the ALK translocated H3122 line even with short courses of ALK inhibition (15 min), while similar cytotoxicity was seen with $72 \mathrm{~h}$ inhibition of PI3K and MEK concurrently (Figure 6), even though both approaches induced major inhibition of phosphorylated AKT and ERK in Western blots after $6 \mathrm{~h}$ treatments (Figure 3A).

Since the results showed that dual inhibition needed to be administered for longer periods of time for maximal

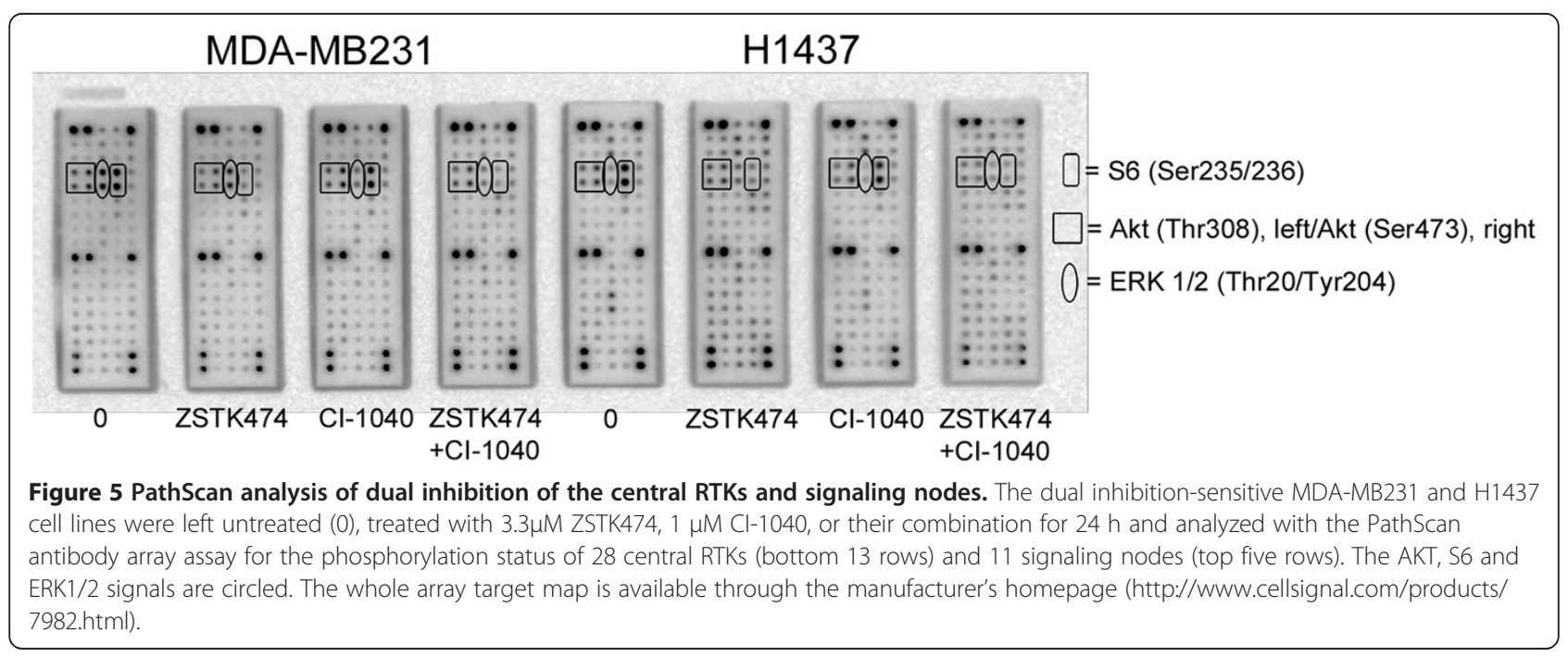




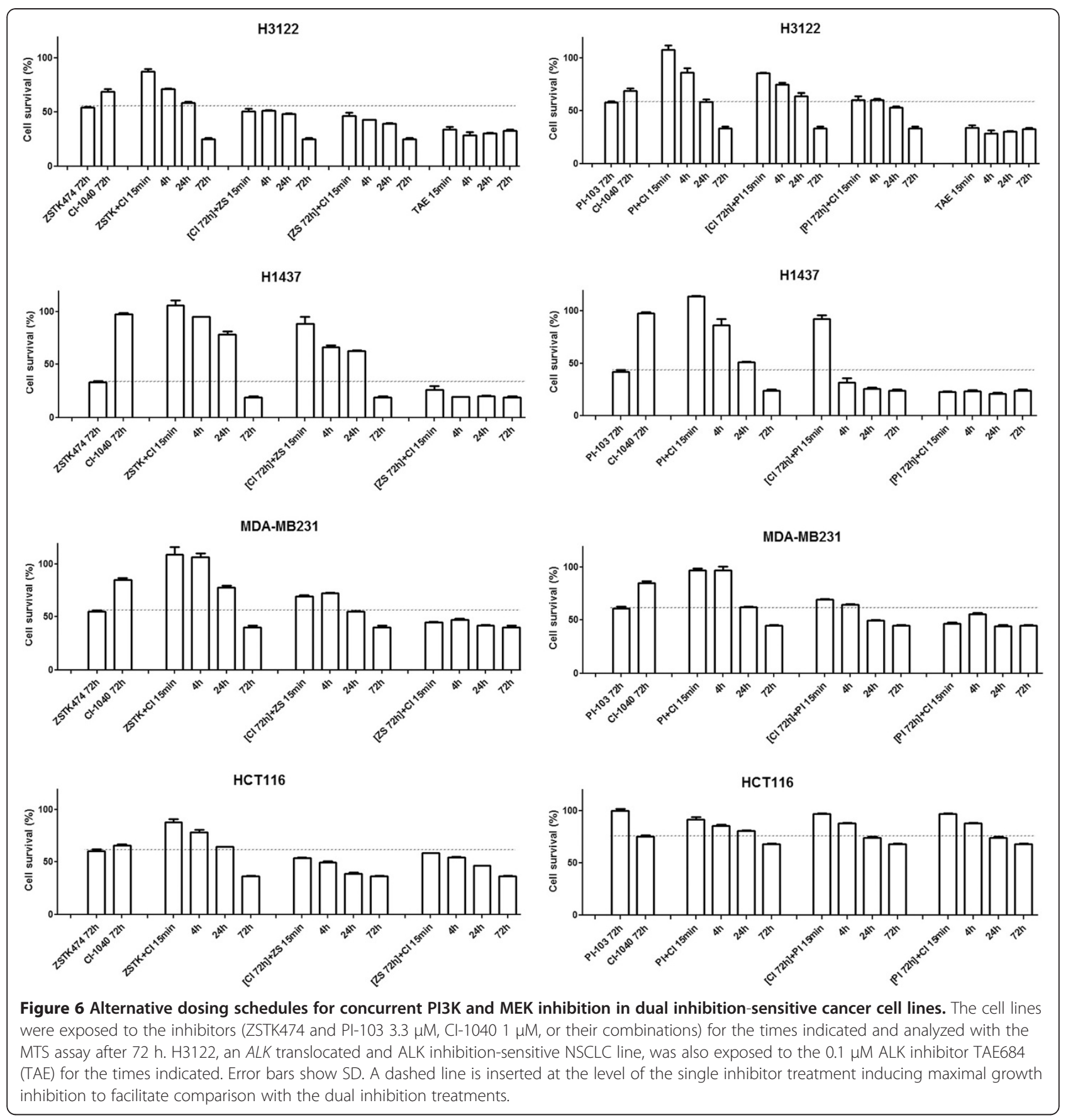

cytotoxicity, we turned next to investigating whether both inhibitors are required throughout the period of exposure. The dual inhibition-sensitive cell lines were exposed to one inhibitor throughout the treatment period (72 h) while the other inhibitor was administered concurrently for $15 \mathrm{~min}, 4 \mathrm{~h}$ or $24 \mathrm{~h}$ at the beginning of the drug exposure. The results varied significantly between the cell lines tested. In the H1437 and MDA-MB231 lines concurrent inhibition of PI3K and MEK for 15 min with continued PI3K inhibition for $72 \mathrm{~h}$ achieved similar cytotoxicity to concurrent inhibition for $72 \mathrm{~h}$ (Figure 6). Conversely, when these lines were exposed to the MEK inhibitor throughout the treatment period, short concurrent exposures (15 $\mathrm{min}, 4 \mathrm{~h}$ or $24 \mathrm{~h}$ ) to PI3K inhibitors did not induce any comparable cytotoxicity (Figure 6). On the other hand, the effects of dual inhibition with PI-103 occurred faster in the H1437 line than with ZSTK474, since shorter exposures to the drug $(24 \mathrm{~h})$ seemed to be sufficient for maximal cytotoxicity as compared with $72 \mathrm{~h}$ of ZSTK474 (Figure 6). In the case of the H3122 and HCT116 lines, 
both the PI3K and MEK inhibitors needed to be administered throughout the treatment period for maximal cytotoxicity (Figure 6).

We next investigated alternative dosing of the dual inhibition of cell signaling. The dual inhibition-sensitive lines were exposed to the PI3K inhibitors and MEK inhibitor concurrently for $15 \mathrm{~min}$, after which treatment was continued with a single inhibitor for the remainder of the $6 \mathrm{~h}$ period. pAKT downregulation was complete or nearly complete when the cells were treated for only $15 \mathrm{~min}$ and with PI3K inhibitors for $6 \mathrm{~h}$ (Figure 3B), while conversely, pERK $1 / 2$ recovered completely in $6 \mathrm{~h}$ when the cells were treated with the MEK inhibitor for $15 \mathrm{~min}$ (Figure 3B). Interestingly, we were able to see some recovery in the activity of the downstream targets of AKT when the PI3K inhibitors were administered for $15 \mathrm{~min}$ despite the remaining pAKT downregulation. The pS6 signal was able to recovery in the MDA-MB231 (with ZSTK474) and HCT116 (with both PI3K inhibitors) lines after short PI3K administration (Figure 3B). Furthermore, p4E-BP1 recovery was noted in the H3122 (with ZSTK474), MDA-MB231 (with ZSTK474), and HCT116 (with both PI3K inhibitors) lines (Figure 3B). Interestingly, MEK inhibitor treatment induced upregulation of p4E-BP1 in the MDA-MB231 line (Figure 3A), and marked downregulation p4E-BP1 was noted only with PI-103 (PI3K and mTOR inhibitor) in the alternative dosing experiments, but not with ZSTK474 (with a PI3K inhibitor alone) (Figure 5), suggesting mTORmediated activation of 4E-BP1 in response to MEK inhibition. TAE684, an ALK inhibitor, treatment was also included in the experiments conducted with the H3122 line, and this induced comparable pAKT, pERK1/2, and pS6 downregulation to that achieved with dual inhibition, whereas no change in p4E-BPI was noted (Figure 3B). Some recovery of pAKT and pS6 was seen after a short treatment with TAE684 (Figure 3B).

We went on further to analyze whether the alternative dosing could also result in apoptosis in the H3122 cell line, the only line identified as inducing apoptosis in response to dual inhibition. When the cells was treated for $15 \mathrm{~min}$ with dual inhibition and treatment with either the PI3K inhibitors or the MEK inhibitor was continued for $48 \mathrm{~h}$, marked PARP cleavage was seen in all the treatments (Figure 4B). Furthermore, 15 min treatment with an ALK inhibitor resulted in marked PARP cleavage (Figure 4B). Cleaved PARP results were further verified with western blot analysis for cleaved caspase-3, another marker for apoptosis. Cleaved caspase-3 was detected with concurrent PI3K and MEK, or ALK inhibition while no signal was seen in PI3K or MEK inhibitor treatments. Conversely to cleaved PARP, the cleaved caspase- 3 signal was much lower in alternative dosing schedules compared to continuous, concurrent PI3K and MEK inhibition (Figure 4C).

\section{Discussion}

The PI3K-AKT and RAS-RAF-MEK-ERK signaling pathways are thought to be the central mediators of oncogenic signals in solid malignancies. Multiple inhibitors targeting PI3K, AKT, RAF and MEK are under development for cancer therapy, but early-phase clinical trials suggest that the single agent efficiency of such inhibitors seems to be limited, except in the case of the Raf mutant melanoma, where both RAF and MEK inhibitors have high clinical activity. There is preclinical evidence that combining the inhibitors of both pathways provides more efficient cancer therapy [2,4,6,7], and some earlyphase clinical trials are under way to test this approach.

We investigated here the dual pharmacological inhibition of PI3K and MEK in NSCLC cell line models with specific oncogenic genotypes. All the cell lines tested were highly responsive to single-agent PI3K inhibitors, showing a strong correlation with maximal target inhibition. This suggests that the PI3K-AKT pathway has a central role in transmitting oncogenic signals from various upstream sources, and therefore the responses to pathway inhibition are not limited to any specific cancer genotype. Furthermore, the data suggest a central role for pathway activation in the proliferation of carcinomas. The cytotoxicity of PI3K inhibitors seemed to be comparable when a PI3K (ZSTK474) or PI3K/mTOR (PI103) inhibitors alone were used, suggesting that only PI3K inhibition matters for cytotoxicity, as administration of the MEK inhibitor seemed to have limited activity or none at all in the models tested. Two out of the twelve cell lines tested showed significantly increased cytotoxicity in response to the concurrent administration of PI3K and MEK inhibitors. Analogously to previous studies, the activity of dual inhibition was not associated with any specific oncogenic genotype, since ALK translocation-positive (H3122) and triple-negative (H1437) cell lines were the most responsive ones [6]. In MEK inhibition-sensitive models. such as triple-negative breast or K-Ras mutant colorectal cancers have shown additive cytotoxicity or reversal of resistance when MEK inhibitors have been combined with inhibitors of the PI3K-AKT-mTOR pathway [4,7]. It is interesting to note that the dual inhibition-sensitive NSCLC lines identified here showed some cytotoxicity in response to low concentrations of MEK inhibitors $(<1 \mu \mathrm{M})$, thereby differing from the other lines tested, which showed no response or a response only to high concentrations of the inhibitor. Furthermore, the K-Ras, EGFR and ALK wild-type cell $\mathrm{H} 1437$ is of a rare oncogenic genotype, a MEK1 mutant, and has previously been identified as being sensitive to MEK inhibitor treatment alone [21]. Based on the current data and previously reported findings, one could speculate that dual PI3K and MEK inhibition therapy could be the most efficient for cancers that show some 
dependence on MEK signaling for their proliferation or survival. Mechanistically, sensitivity to dual PI3K and MEK inhibition remains to be elucidated. It is likely that the responses are not associated with any specific oncogenic genotype but rather with inhibition of the effects of feedback activation induced by the inhibition of one pathway on the other. If this also holds good in vivo, it is likely to make the selection of patients for such treatment difficult, since no predictive biomarkers of feedback activation exist.

Even though dual inhibition of PI3K-AKT and MEK has been identified as an effective cancer therapy in preclinical models, it questionable whether this therapy is tolerable in a clinical setting concentrations high enough to achieve sufficient target inhibition. Early-phase clinical trials are in progress to test different doses and dosing schedules, but the optimal administration for maximal efficiency and tolerability remains to be elucidated. In the light of recent data from the ASCO 2012 Annual Meeting, PI3K and MEK inhibitor combination treatments are now being tested in concurrent and intermittent schedules [22,23]. The tolerability of intermittent administration may enable higher doses of the agents to be administered than with continuous concurrent treatment [23]. The cell line model data presented here suggest that even short courses of concurrent administration can cause marked cytotoxicity and/or apoptosis. Two out of the four dual inhibition-sensitive cell lines showed comparable cytotoxicity to that achieved with continuous administration of dual inhibition when the MEK inhibitor was administered for short periods (15 min) in combination with continuous PI3K inhibitor treatment. The increased cytotoxicity occurred even though the effects of the MEK inhibitor were quickly reversed $(<6 \mathrm{~h})$ after wash-out of the drug. Meanwhile $\mathrm{H} 3122$, an $A L K$ translocated cell line, showed apoptosis in response to short concurrent administration of the drugs even though longer concurrent administration led to maximal cytotoxicity. Interestingly, short courses of ALK inhibition (15 min) induced comparable cytotoxicity to long administration of either an ALK inhibitor or a dual inhibitor combination, even though the ALK inhibitor is reversible in its mode of action and some recovery of the target inhibition is known to occur within $6 \mathrm{~h}$. In the light of our in vitro data, one could hypothesize that even a short course of dual inhibitor administration could have similar clinical effects with better tolerability. Analogously, a recent work has shown that intermittent administration of concurrent PI3K and MEK inhibition can induce robust growth inhibition in cancer cell lines [24]. Better alternative dosing schedules for achieving clinical tolerability could also enable the use of higher doses of the drugs, leading to stronger inhibition of the target. Short but more significant target inhibition is likely to be more efficient than submaximal inhibition for longer periods. Our data point to the importance of maximal inhibition of the target and a preferential role for longer PI3K-AKT pathway inhibition when dual inhibition is used. These data are based only on in vitro models, however, and correlation with the in vivo situation is not always a straightforward matter.

The interconnectivity of the PI3K-AKT-mTOR and RAS-RAF-MEK-ERK pathways makes the idea of their concurrent dual inhibition an appealing one. The present cell signaling experiments also showed high interconnectivity of these two pathways, since in many instances inhibition of one pathway resulted in concurrent feedback activation of the other. Furthermore, another MEK inhibition-induced feedback mechanism was identified in the MDA-MB231 cell line which led to the activation of 4E-BP1 independently of PI3K-AKT. Previous studies have suggested that the PI3K-AKT-mTOR and RAS-RAF-MEK-ERK pathway signals converge at $4 \mathrm{E}-\mathrm{BP} 1$, and that its inhibition may be a major determinant of the efficiency of dual inhibition [25]. Conversely, we did not find any correlation between the efficiency of dual inhibition and 4E-BP1 downregulation, since the 4E-BP1 signal correlated significantly only with PI3KAKT-mTOR activity and cytotoxicity occurred without it being downregulated. In also, some of the treatment schedules induced marked cytotoxicity in the H3122 and MDA-MB231 cell lines without the induction of any marked 4E-BP1downregulation.

\section{Conclusions}

The most important findings to emerge from this investigation of the concurrent dual inhibition of PI3K and MEK for cancer therapy purposes are the fact that alternative dosing schedules result in comparable cytotoxicity to that achieved with continuous treatment schedules, and that the responses to dual inhibition can be achieved in multiple cancer genotypes. The present preclinical data may offer new leads for clinical progress towards more efficient and tolerable cancer therapies.

\section{Competing interests}

The authors' declare no competing interests.

\section{Authors' contributions}

JPK conceived the study design and was coordinated the work. JPK and EJ carried out the laboratory experiments, participated in the gathering, analysis and interpretation of the data, and drafted, read and approved the final version of the manuscript. All authors read and approved the final manuscript.

\section{Acknowledgements}

We wish to thank Anne Bisi for her technical assistance. This research was supported by the Cancer Society of Northern Finland, the Emil Aaltonen Foundation, the Finnish Foundation for Tuberculosis Resistance, the Finnish Oncological Society, Oulu University Hospital, the Orion-Farmos Science Foundation, and the Sigrid Juselius Foundation. 
Received: 29 March 2012 Accepted: 18 December 2012

Published: 21 December 2012

\section{Refererences}

1. Hanahan D, Weinberg RA: Hallmarks of cancer: the next generation. Cell 2011, 144(5):646-674.

2. Faber AC, Li D, Song Y, Liang MC, Yeap BY, Bronson RT, Lifshits E, Chen Z, Maira SM, Garcia-Echeverria C, et al: Differential induction of apoptosis in HER2 and EGFR addicted cancers following PI3K inhibition. Proc Natl Acad Sci U S A 2009, 106(46):19503-19508.

3. Solit DB, Garraway LA, Pratilas CA, Sawai A, Getz G, Basso A, Ye Q, Lobo JM She $Y$, Osman I, et al: BRAF mutation predicts sensitivity to MEK inhibition. Nature 2006, 439(7074):358-362.

4. Hoeflich KP, O'Brien C, Boyd Z, Cavet G, Guerrero S, Jung K, Januario T, Savage $H$, Punnoose $E$, Truong $T$, et al: In vivo antitumor activity of MEK and phosphatidylinositol 3-kinase inhibitors in basal-like breast cancer models. Clin Cancer Res 2009, 15(14):4649-4664.

5. Chandarlapaty S, Sawai A, Scaltriti M, Rodrik-Outmezguine V, Grbovic-Huezo $\mathrm{O}$, Serra V, Majumder PK, Baselga J, Rosen N: AKT inhibition relieves feedback suppression of receptor tyrosine kinase expression and activity. Cancer Cell 2011, 19(1):58-71.

6. Sos ML, Fischer S, Ullrich R, Peifer M, Heuckmann JM, Koker M, Heynck S, Stuckrath I, Weiss J, Fischer F, et al: Identifying genotype-dependent efficacy of single and combined PI3K- and MAPK-pathway inhibition in cancer. Proc Natl Acad Sci U S A 2009, 106(43):18351-18356.

7. Wee S, Jagani Z, Xiang KX, Loo A, Dorsch M, Yao YM, Sellers WR, Lengauer C, Stegmeier F: PI3K pathway activation mediates resistance to MEK inhibitors in KRAS mutant cancers. Cancer Res 2009, 69(10):4286-4293.

8. O'Reilly KE, Rojo F, She QB, Solit D, Mills GB, Smith D, Lane H, Hofmann F, Hicklin DJ, Ludwig DL, et al: mTOR inhibition induces upstream receptor tyrosine kinase signaling and activates Akt. Cancer Res 2006, 66(3):1500-1508

9. Paez JG, Janne PA, Lee JC, Tracy S, Greulich H, Gabriel S, Herman P, Kaye FJ, Lindeman N, Boggon TJ, et al: EGFR mutations in lung cancer: correlation with clinical response to gefitinib therapy. Science 2004, 304(5676):1497-1500.

10. Shaw AT, Yeap BY, Mino-Kenudson M, Digumarthy SR, Costa DB, Heist RS, Solomon B, Stubbs H, Admane S, McDermott U, et al: Clinical features and outcome of patients with non-small-cell lung cancer who harbor EML4ALK. J Clin Oncol 2009, 27(26):4247-4253.

11. Soda M, Choi YL, Enomoto M, Takada S, Yamashita Y, Ishikawa S, Fujiwara S, Watanabe $H$, Kurashina $\mathrm{K}$, Hatanaka $\mathrm{H}$, et al: Identification of the transforming EML4-ALK fusion gene in non-small-cell lung cancer. Nature 2007, 448(7153):561-566.

12. Koivunen JP, Mermel C, Zejnullahu K, Murphy C, Lifshits E, Holmes AJ, Choi HG, Kim J, Chiang D, Thomas R, et al: EML4-ALK fusion gene and efficacy of an ALK kinase inhibitor in lung cancer. Clin Cancer Res 2008, 14(13):4275-4283.

13. Mok TS, Wu YL, Thongprasert S, Yang CH, Chu DT, Saijo N, Sunpaweravong $P$, Han B, Margono B, Ichinose $Y$, et al: Gefitinib or carboplatin-paclitaxel in pulmonary adenocarcinoma. New Engl J Med 2009, 361(10):947-957.

14. Kwak EL, Bang YJ, Camidge DR, Shaw AT, Solomon B, Maki RG, Ou SH, Dezube BJ, Janne PA, Costa DB, et al: Anaplastic lymphoma kinase inhibition in non-small-cell lung cancer. New Engl J Med 2010, 363(18):1693-1703.

15. Adjei AA: K-ras as a target for lung cancer therapy. J Thorac Oncol 2008, 3(6 Suppl 2):S160-S163.

16. Sebolt-Leopold JS, Dudley DT, Herrera R, Van Becelaere K, Wiland A, Gowan RC, Tecle H, Barrett SD, Bridges A, Przybranowski S, et al: Blockade of the MAP kinase pathway suppresses growth of colon tumors in vivo. Nat Med 1999, 5(7):810-816.

17. Yaguchi S, Fukui Y, Koshimizu I, Yoshimi H, Matsuno T, Gouda H, Hirono S, Yamazaki K, Yamori T: Antitumor activity of ZSTK474, a new phosphatidylinositol 3-kinase inhibitor. J Natl Cancer Inst 2006, 98(8):545-556.

18. Kong D, Yaguchi S, Yamori T: Effect of ZSTK474, a novel phosphatidylinositol 3-kinase inhibitor, on DNA-dependent protein kinase. Biol Pharm Bull 2009, 32(2):297-300.

19. Bagci-Onder T, Wakimoto $H$, Anderegg M, Cameron C, Shah K: A dual PI3K/ mTOR inhibitor, PI-103, cooperates with stem cell-delivered TRAIL in experimental glioma models. Cancer Res 2011, 71(1):154-163.
20. Zou ZQ, Zhang XH, Wang F, Shen QJ, Xu J, Zhang LN, Xing WH, Zhuo RJ, Li D: A novel dual PI3Kalpha/mTOR inhibitor PI-103 with high antitumor activity in non-small cell lung cancer cells. Int J Mol Med 2009, 24(1):97-101.

21. Marks JL, Gong Y, Chitale D, Golas B, McLellan MD, Kasai Y, Ding L, Mardis ER, Wilson RK, Solit D, et al: Novel MEK1 mutation identified by mutational analysis of epidermal growth factor receptor signaling pathway genes in lung adenocarcinoma. Cancer Res 2008, 68(14):5524-5528.

22. Bedard P, Tabernero J, Kurzrock R, Britten C, Stathis A, Perez-Garcia J, Zubel A, Le N, Carter K, Bellew K, et al: A phase Ib, open-label, multicenter, doseescalation study of the oral pan-PI3K inhibitor BKM120 in combination with the oral MEK1/2 inhibitor GSK1120212 in patients (pts) with selected advanced solid tumors. J Clin Oncol 2012, 30(15_Suppl 1):3003.

23. LoRusso P, Shapiro G, Panday S, Kwak E, Jones C, Belvin M, Musib L, de Crespigny A, McKenzie M, Gates M, et al: A first-in-human phase Ib study to evaluate the MEK inhibitor GDC-0973, combined with the pan-PI3K inhibitor GDC-0941, in patients with advanced solid tumors. J Clin Oncol 2012, 30(15_Suppl 1):2566.

24. Hoeflich KP, Merchant M, Orr C, Chan J, Den Otter D, Berry L, Kasman I, Koeppen H, Rice K, Yang NY, et al: Intermittent administration of MEK inhibitor GDC-0973 plus PI3K inhibitor GDC-0941 triggers robust apoptosis and tumor growth inhibition. Cancer Res 2012, 72(1):210-219.

25. She QB, Halilovic E, Ye Q, Zhen W, Shirasawa S, Sasazuki T, Solit DB, Rosen $\mathrm{N}$ : 4E-BP1 is a key effector of the oncogenic activation of the AKT and ERK signaling pathways that integrates their function in tumors. Cancer Cell 2010, 18(1):39-51.

doi:10.1186/1471-2407-12-612

Cite this article as: Jokinen et al:: Alternative dosing of dual PI3K and MEK inhibition in cancer therapy. BMC Cancer 2012 12:612.

\section{Submit your next manuscript to BioMed Central and take full advantage of:}

- Convenient online submission

- Thorough peer review

- No space constraints or color figure charges

- Immediate publication on acceptance

- Inclusion in PubMed, CAS, Scopus and Google Scholar

- Research which is freely available for redistribution

Submit your manuscript at www.biomedcentral.com/submit
C) Biomed Central 